\title{
Decentralized Model Reference Control of Flexible Cable-Stayed Beam Structures *
}

\author{
Ningsu Luo, Dept. of Electronics, Automation and Computer Engineering, \\ University of Girona, 17071 Girona, SPAIN. ningsu@eia.udg.es \\ José Rodellar, Dept. of Applied Mathematics III, Technical University \\ of Catalonia, 08034 Barcelona, SPAIN. rodellar@etseccpb.upc.es \\ Manuel De la Sen, Dept. of Electricity and Electronics, University of \\ Basque Country, 48940 Leioa, SPAIN. msen@we.lc.ehu.es \\ Josep Vehí, Dept. of Electronics, Automtion and Computer Engineering, \\ University of Girona, 17071 Girona, SPAIN. vehi@eia.udg.es
}

\begin{abstract}
In this paper, a decentralized model reference controller is designed to reduce the magnitude of the transversal vibration of a flexible cable-stayed beam structure induced by a seismic excitation. The controller design is made based on the principle of sliding mode such that $a$ priori knowledge on the bounds of the uncertainties and disturbances is not requried. A numerical simulation example is presented to illustrate the effectiveness of the proposed control scheme for a scaled model of bridge.
\end{abstract}

\section{Introduction}

In the recent years, active and semi-active control schemes have been proposed for the protection of cablestayed bridge structures in the presence of external forces such as traffic, heavy winds and earthquakes [1]. Among them, the centralized linear active control ${ }^{[2]}$ and decentralized nonlinear active control ${ }^{[3]}$ have received an important attention. In this paper, the dynamic behaviour of this particular type of beam structure is characterized by a nonlinear mathematical model with interconnection terms ${ }^{[3]}$. A practical decentralized active controller is presented to attenuate the transversal vibration of a flexible cable-stayed bridge structure induced by seismic excitation, in which the knowledge on the exact value of system parameters, structural disturbances and the seismic excitation is not required a priori in the controller design.

${ }^{*}$ Research supported in part by the Spanish Government through the grants PB93-0005, PB93-1040 and PB96-0257 and NATO collaborative research grant CRG 971534

$0-7803-4990-6 / 99 \$ 10.00$ ○ 1999 AACC

\section{Problem Formulation}

Cable-stayed bridge is flexible beam structure with very small structural damping. In the active control systems, sensors are put at the anchor points of the stayed cables to measure the relative vertical displacements and velocities of the bridge deck, respectively. The control forces are generated by the actuators to increase or decrease the effective length of the stay cables. The technique of finite element has been used for modelling this particular bridge structure ${ }^{[3]}$. It is assumed that: (a) The mass of the cable that affects degree of freedom $i$ is small compared to the $i$ th concentrated mass of such a degree of freedom; (b) The stay cables are used as active tendons and operate in the linear elastic regime. Their sags are minimal and therefore neglected. (c) The structural damping is very small and can be neglected. Denote $x_{i}^{d}(t), x_{i}^{v}(t)$ as the relative transversal displacement and velocity of the bridge deck, respectively, measured at the location where the $i t h$ stay cable is anchored, and $x_{i}(t)=\left[x_{i}^{d}(t), x_{i}^{v}(t)\right]^{T}$ as the state vector $(i=1, \cdots, N)$. Then, the dynamic behaviour of the bridge segment, accounting for the effect of the stay cables, is described by the following mathematical model being decomposed into $N$ subsystems $S_{i}$ :

$\dot{x}_{i}(t)=\boldsymbol{A}_{i} \boldsymbol{x}_{i}(t)+\boldsymbol{b}_{i}(t) u_{i}(t)+\boldsymbol{d}_{i}\left[f_{n i}(t)+f_{d i}(t)+f_{e i}(t)\right]$

$$
\begin{gathered}
\boldsymbol{A}_{i}=\left(\begin{array}{cc}
0 & 1 \\
-k_{i i} / m_{c} & 0
\end{array}\right) ; \boldsymbol{d}_{i}=\left(\begin{array}{c}
0 \\
1 / m_{c}
\end{array}\right) \\
\boldsymbol{b}_{i}(t)=\left(\begin{array}{c}
0 \\
-c_{\boldsymbol{a} i} c_{e i} \sin \theta_{i}(t) / m_{\boldsymbol{c}} c_{l i}
\end{array}\right)
\end{gathered}
$$




$$
\begin{gathered}
f_{n i}(t)=c_{a i} c_{e i}\left[\sin \theta_{i}(t)-\sin \theta_{p i}\right] \\
f_{d i}(t)=-\sum_{\substack{j=1 \\
j \neq i}}^{N}\left(k_{i j}, 0\right)^{T} x_{j}(t)+h_{i}[x(t)] \\
f_{e i}(t)=m_{c} a_{q k}(t)
\end{gathered}
$$

where $x(t)=:\left[x_{1}(t), \cdots, x_{N}(t)\right]^{T} . \quad m_{c}$ is the concentrated mass and $k_{i j}(i, j=1, \cdots, N)$ are stiffness parameters of the controlled concentrated masses that include the effect of the stay cables. Positive scalars $c_{a i}$, $c_{e i}$ and $c_{l i}$ are the cross-sectional area, Young modulus of elasticity and unstressed length, respectively, of the ith stay cable. $u_{i}(t)$ is a control force applied to the ith stay cable to increase or decrease the its effective length. The scalar function $f_{n i}(t)$ describes the nonlinearities due to the cable-beam geometry. $\theta_{i}(t)$ and $\theta_{p i}$ are the stressed and prestressed angles between the $i t h$ stay cable and the local horizontal at the point where the stay cable is anchored to the bridge deck, respectively. The unknown scalar function $h_{i}[\boldsymbol{x}(t)]$ represents the structural disturbances or the influence of residual modes appeared at the anchor points and the scalar function $\sum_{\substack{j=1 \\ j \neq i}}^{N}\left(k_{i j}, 0\right)^{T} \boldsymbol{x}_{j}(t)$ describes the interconnection effects among the local subsystems. $a_{q k}(t)$ is the vertical component of an unknown earthquake force.

Assumption 1. The system parameters have upper and lower bounds such that $m_{c} \in\left[m_{c}^{-}, m_{c}^{+}\right], c_{a i} \in$ $\left[c_{a i}^{-}, c_{a i}^{+}\right], c_{e i} \in\left[c_{e i}^{-}, c_{e i}^{+}\right], c_{l i} \in\left[c_{l i}^{-}, c_{l i}^{+}\right]$and $k_{i j} \in\left[k_{i j}^{-}, k_{i j}^{+}\right]$ with $c_{a i}^{-}, c_{a i}^{+}, c_{e i}^{-}, c_{e i}^{+}, c_{l i}^{-}, c_{l i}^{+}, m_{c}^{-}, m_{c}^{+}, k_{i j}^{-}, k_{i j}^{+}(i, j=$ $1, \cdots, N)$ being known positive constants, respectively.

Assumption 2. The vertical component of an unknown earthquake acceleration to the bridge deck $a_{q k}(t)$ is uniformly bounded such that $a_{q k}(t) \in\left[a_{q k}^{-}, a_{q k}^{+}\right]$for all $t \geq 0$ with $a_{q k}^{-}$and $a_{q k}^{+}$being known non-zero constants.

Assumption 3. The angle between the ith stay cable and the local horizontal at the anchor points is uniformly bounded such that $\theta_{i}(t), \theta_{p i} \in\left[\theta_{i}^{-}, \theta_{i}^{+}\right](i=1, \cdots, N)$ for all $t \geq 0$ with $\theta_{i}^{-}$and $\theta_{i}^{+}$being known positive real numbers that lie in the range $(0, \pi / 2)$ radians.

Assumption 4. $f_{d i}(t)(i=1, \cdots, N)$ are unknown scalar functions that represent the unknown structural disturbances appeared at the anchor point of the ith stay cable and the interconnection effects among the local subsystems such that the following relationships hold:

$$
\left|f_{d i}(t)\right| \leq \alpha_{i}+\sum_{j=1}^{N} \beta_{i j}\left\|\boldsymbol{x}_{j}(t)\right\|
$$

where $\alpha_{i}$ and $\beta_{i j}(i, j=1, \cdots, N)$ are known nonnegative constants.

\section{Active Control System}

The main objective of an active control system is to improve the dynamic performance of a bridge structure in the presence of traffic, heavy wind and seismic excitation, which is usually difficult to obtain by using only a passive control system. In the recent years, the active protection of civil engineering structures via sliding mode control has received a great attention due to its excellent robustness properties to the parametric uncertainties and external disturbances $[4]-[5]$. In this paper, the principle of sliding mode control ${ }^{[6]}$ is extended to the decentralized model reference control in order to attenuate the transversal vibration of a cable-stayed bridge caused by the vertical component of an unknown earthquake.

\subsection{Reference model}

First, suppose that the desired behaviour of the ith subsystem $S_{i}$ is described by a linear exponentially stable reference subsystem $S_{m i}$ defined below, which has the same dimension as the corresponding controlled subsystem $S_{i}(i=1, \cdots, N)$.

$$
\begin{gathered}
S_{m i}: \quad \dot{\boldsymbol{x}}_{m i}(t)=\boldsymbol{A}_{m i} \boldsymbol{x}_{m i}(t)+\boldsymbol{b}_{m i} r_{i}(t) \\
\boldsymbol{A}_{m i}=\left(\begin{array}{cc}
0 & 1 \\
-\omega_{m}^{2} & -2 \zeta_{m} \omega_{m}
\end{array}\right) ; \boldsymbol{b}_{m i}(t)=\left(\begin{array}{c}
0 \\
\omega_{m}^{2}
\end{array}\right)
\end{gathered}
$$

where $x_{m i}(t)=:\left[x_{m i}^{d}(t), x_{m i}^{v}(t)\right]^{T}$ with $x_{m i}^{d}(t)$ and $x_{m i}^{v}(t)$ being the desired transversal relative displacement and velocity of the local reference model, $\zeta_{m}$ the desired damping factor and $\omega_{m}$ the desired natural frequency for the controlled cable-stayed bridge structure and $r_{i}(i=1, \cdots, N)$ are reference inputs $\left(r_{i}(t) \equiv 0\right.$ in this case), respectively. Define the tracking error vector $\boldsymbol{e}_{i}(t)=:\left[e_{i}^{d}(t), e_{i}^{v}(t)\right]^{T}$ as follows:

$$
e_{i}^{d}(t)=: x_{m i}^{d}(t)-x_{i}^{d}(t) ; e_{i}^{v}(t)=: x_{m i}^{v}(t)-x_{i}^{v}(t)
$$

Then, the following tracking error dynamic subsystems $S_{e i}(i=1, \cdots, N)$ are obtained:

$$
S_{e i}: \dot{\boldsymbol{e}}_{i}(t)=\boldsymbol{A}_{m i} \boldsymbol{e}_{i}(t)+\left(\boldsymbol{A}_{m i}-\boldsymbol{A}_{i}\right) \boldsymbol{x}_{i}(t)
$$




$$
-\boldsymbol{b}_{i}(t) u_{i}(t)-\boldsymbol{d}_{i}\left[f_{n i}(t)+f_{d i}(t)+f_{e i}(t)\right]
$$

A model reference sliding mode control scheme is presented below to drive the tracking errors to zero asymptotically and thus the desired performance, with the damping factor $\zeta_{m}$ and natural frequency $\omega_{m}$, is achieved in the controlled cable-stayed bridge structure.

\subsection{Decentralized controller design}

In the controller design, the following sliding function $\sigma_{i}(t) \in \mathbb{R}$ is defined for the tracking error dynamic subsystem $S_{e i}(i=1, \cdots, N)$

$$
\sigma_{i}(t)=\boldsymbol{\epsilon}_{i}^{T} \boldsymbol{e}_{i}(t)=\epsilon_{d i} e_{i}^{d}(t)+\epsilon_{v i} e_{i}^{v}(t)
$$

where $\epsilon_{i}=:\left[\epsilon_{d i}, \epsilon_{v i}\right]^{T}$, with $\epsilon_{d i}>0$ and $\epsilon_{v i}>0$, is a parameter vector to be chosen in order to guarantee the closed-loop asymptotic stability of the tracking error dynamic subsystem $S_{e i}$. When $\sigma_{i}(t)=0(i=1, \cdots, N)$ for $t \geq t_{s}$, it is said that sliding motion is generated in the tracking error dynamic subsystems $S_{e i}(i=1, \cdots, N)$. Denote

$$
\eta_{i}(t)=:-\boldsymbol{\epsilon}_{i}^{T} \boldsymbol{b}_{i}(t)
$$

then from the definition of $\boldsymbol{b}_{i}(t)$, it is easy to show that

$$
\eta_{i}(t) \geq \eta_{i}^{*}>0 \quad ; \quad \eta_{i}^{*}=\frac{\epsilon_{i v} c_{a i}^{-} c_{e i}^{-} \sin \theta_{i}^{-}}{m_{c}^{+} c_{l i}^{+}}
$$

Since the reference model is exponentially stable, one has $x_{m i}(t) \rightarrow 0$ as $t \rightarrow \infty$. Thus, under Assumptions $1-4$, the following relationship holds for all $t \geq 0$

$$
\begin{gathered}
\left|\boldsymbol{\epsilon}_{i}^{T}\left(\boldsymbol{A}_{m i}-\boldsymbol{A}_{i}\right) \boldsymbol{x}_{i}(t)-\boldsymbol{\epsilon}_{i}^{T} \boldsymbol{d}_{i}\left[f_{n i}(t)+f_{d i}(t)+f_{e i}(t)\right]\right| \\
\leq p_{i 0}+\sum_{j=1}^{N} p_{i j}\left\|\boldsymbol{e}_{j}(t)\right\|
\end{gathered}
$$

where $p_{i 0}, p_{i j}(i, j=1, \cdots, N)$ are some known nonnegative constants.

The global reaching condition of a sliding mode is $\sum_{i=1}^{N} c_{i}(t) \sigma_{i}(t) \dot{\sigma}_{i}(t)<0$, where $c_{i}(t)=c_{i}^{*} /\left|\sigma_{i}(t)\right|$ for $\sigma_{i}(t) \neq 0$ and $c_{i}(t)=0$ for $\sigma_{i}(t)=0$ with $c_{i}^{*}(i=1, \ldots N)$ being some positive constants. The following decentralized model reference controller is proposed

$$
u_{i}(t)=-\psi_{i \sigma} \sigma_{i}(t)-\left[\psi_{i o}+\psi_{i e}\left\|\boldsymbol{e}_{i}(t)\right\|\right] \operatorname{sgn}\left[\sigma_{i}(t)\right]
$$

where $\psi_{i \sigma}, \psi_{i o}, \psi_{i e}(i=1, \cdots, N)$ are positive constant parameters to be chosen by the designer.
Theorem 1. Under Assumptions 1-4, sliding motion is generated in the tracking error dynamic subsystems $S_{\mathrm{e} i}$ $(i=1, \cdots, N)$ by using the decentralized model reference sliding mode controller (7) and the tracking error vectors $\boldsymbol{e}_{i}(t)(i=1, \cdots, N)$ are bounded for all $t \geq 0$ and converge to zero asymptotically as $t \rightarrow \infty$ if the following relationships hold

$$
\begin{gathered}
\psi_{i \sigma}=\left(\eta_{i}^{*}\right)^{-1} \psi_{\sigma}>0 \\
\psi_{i o}>\left(\eta_{i}^{*}\right)^{-1} p_{i 0} \\
\psi_{i e}>\left(\eta_{i}^{*}\right)^{-1}\left[\left\|\boldsymbol{\epsilon}^{T} \boldsymbol{A}_{m i}\right\|+p_{i i}+\sum_{\substack{j=1 \\
j \neq i}}^{N} p_{i j}\right]
\end{gathered}
$$

Remark. In the implementation of control law, nonideal effects may cause high control activity which can excite sometimes high-frequency unmodelled dynamics in the system. The chattering phenomenon can be alleviated usually by the using the following decentralized controller:

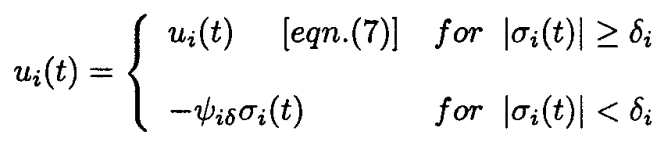

where $\delta_{i}(i=1, \ldots N)$ are small positive constants related to the toleration limit of the dynamic behaviour of the bridge structure and $\psi_{i \delta}(i=1, \ldots, N)$ are non-negative controller gains chosen by the designer. In particular, one can choose $\psi_{i \delta}=0(i=1, \ldots, N)$ to get a practical decentralized controller, in which the active control system is only put into operation when the control performance is degraded below the toleration limit.

\section{Simulation}

The following scale model of the bridge has been used in the simulation: beam (material: steel) with $L=3 \mathrm{~m}$, $A=2 \times 10^{-3} \mathrm{~m}^{2}, E=2.06 \times 10^{11} \mathrm{~N} / \mathrm{m}^{2}, I=$ $6.7 \times 10^{-8} \mathrm{~m}^{4}, m_{c}=7.86 \mathrm{Kg}, k_{11}=1.5596 \times 10^{6} \mathrm{~N} / \mathrm{m}$, $k_{12}=k_{21}=-1.1735 \times 10^{6} \mathrm{~N} / \mathrm{m}, k_{22}=1.5957 \times$ $10^{6} \mathrm{~N} / \mathrm{m}, \rho=7860 \mathrm{Kg} / \mathrm{m}^{3}$ and cables (material: stainless steel wires, SUS304JIS) with $c_{l 1}=3.58 \mathrm{~m}$, $c_{l 2}=3.327 \mathrm{~m}, c_{a 1}=c_{a 2}=2.1 \times 10^{-7} \mathrm{~m}^{2}, c_{e 1}=c_{e 2}=$ $1.706 \times 10^{11} \mathrm{~N} / \mathrm{m}^{2}, \theta_{p 1}=56.04^{\circ}, \theta_{p 2}=63.2^{\circ}$. The seismic excitation has been the scaled Taft earthquake. A reference model has been chosen with $\zeta_{m}=\omega_{m}=1.0$, $x_{m 1}^{d}(0)=x_{m 2}^{d}(0)=0.01$. The upper and lower bounds for system parameters corresponding to the Assumptions $1-4$ are defined as the values with $\pm 10 \%$ variation respect to their nominal ones. Also, $\theta_{1}^{-}=\theta_{2}^{-}=40^{\circ}$ and 
$\theta_{1}^{+}=\theta_{2}^{+}=80^{\circ}$ and $p_{10}=p_{2 o}=1000, p_{11}=2.3 \times 10^{5}$, $p_{12}=p_{21}=1.8 \times 10^{5}, p_{22}=2.6 \times 10^{5}$ in (6). The continuous active decentralized controller (11) has been used with $\epsilon_{d i}=3, \epsilon_{v i}=1, \eta_{1}^{*}=548, \eta_{2}^{*}=590, \psi_{1 \sigma}=0.182$, $\psi_{2 \sigma}=0.169, \psi_{1 o}=\psi_{2 o}=2, \psi_{1 e}=\psi_{2 e}=900$, $\delta_{1}=\delta_{2}=0.001, \psi_{1 \delta}=\psi_{2 \delta}=0$. The time histories of the displacements of the bridge deck in presence of the scaled earthquake excitation, without any control and with the active decentralized control, are shown in Figures 2 and 3 , respectively.

\section{Conclusions}

In this paper, a decentralized model reference controller has been designed to reduce the transversal vibration of a cable-stayed bridge segment induced by the seismic excitation. In the controller design, only local sensor information has been used to generate the control signal that is sent to the actuator of the controlled subsystem. The closed-loop robust stability has been achieved through the generation of a sliding motion in the system. It is shown by the numerical simulation that the magnitude of the transversal vibrations of the bridge deck induced by a seismic excitation has been significantly reduced by using the proposed active decentralized controller compared with the uncontrolled case.

\section{References}

[1] Achkire, Y., Preumont, A. (1996), "Active tendon control of cable-stayed bridges", Earthquake Engineering anả Structural Dynamics, 25, pp. 585-597.

[2] Oshumi, A., Sawada, T. (1993), "Active control of flexible structures subject to distributed and seismic disturbances", Journal of Dynamic Systems, Measurements and Control, 115, pp. 649-657.

[3] Magaña, M.E., Rodellar, J. (1998), "Nonlinear decentralized active tendon control of cable-stayed bridges", Journal of Structural Control, 5, 1, pp. $45-62$.

[4] Yang, J.N., J.C. Wu, A.K. Agrawal (1995), "Sliding mode control of seismically excited linear structures", Journal of Engineering Mechanics, ASCE, 121, 1386-1390.

[5] Luo, N., Rodellar, J., De la Sen, M. (1998), "Composite robust active control of seismically excited structures with actuator dynamics", Earthquake Engineering and Structural Dynamics, 27, 3, pp. 301-311.

[6] Utkin, V.I. (1992), Sliding modes in control and optimization, Springer-Verlag, Berlin, Heidelberg.

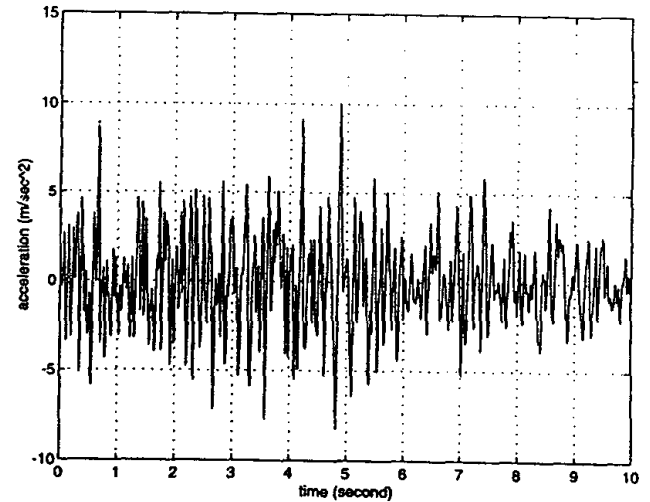

Figure 1. Scaled accelerogram of the vertical component of Taft earthquake

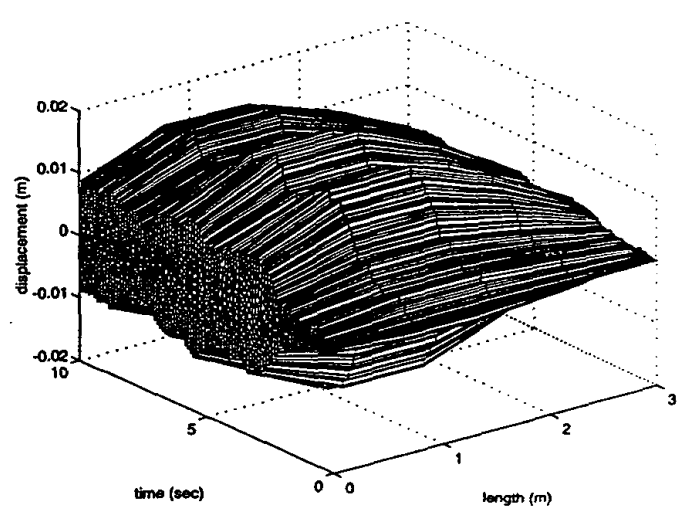

Figure 2. Time response of the bridge without any control

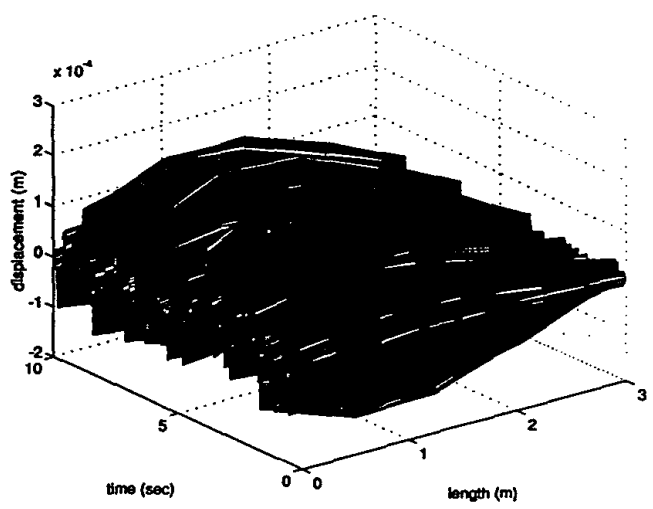

Figure 3. Time response of the bridge with decentralized control 